\title{
ROLE OF COMPONENTS OF MICRORNA MACHINERY IN CARCINOGENESIS
}

\author{
R. Kian, S. Moradi, S. Ghorbian* \\ Department of Molecular Genetics, Ahar Branch, Islamic Azad University, Ahar 54511, Iran
}

\begin{abstract}
MicroRNAs (miRNAs) are a broad class of non-coding RNAs nearly 21 nucleotides length, which play crucial functions in posttranscriptional gene regulation. These molecules are associated with many developmental and cellular processes in eukaryotic organisms. Current investigation has reported major factors contributing to miRNA biogenesis and has constituted basic principles of miRNA function. More recently, it was confirmed that various miRNAs are clearly implicated in human malignancies, such as lung, breast, ovarian, bladder, colon cancer and other kinds of carcinoma. In addition, dysregulation in the miRNA machinery elements such as Dicer, Drosha, DGCR8, Argonaut, and TRBP could be involved in the progress of many tumor types. The purpose of the current review was to compile growing information besides how miRNA biogenesis and gene silencing are modified to develop cancer. Key Words: DGCR8, miRNA machinery components, miR, cancer, regulator.
\end{abstract}

Several types of short non-coding RNAs including small interfering RNAs (siRNAs), Piwi-interacting RNAs (piRNAs) and microRNAs (miRNAs) have a principal and crucial regulatory functions of development processes in all eukaryotes [1]. Formerly, a finding revealed the first miRNAs in Caenorhabditis elegans [2]. miRNAs are extremely protected from species and supposedly may regulate up to $30 \%$ of all genes in the human genome $[3,4]$. miRNAs are small noncoding RNAs of 18-24 nt in length that controls gene expression post-transcriptionally [5] via negative regulation through binding to the 3 '-untranslated region (3'-UTR) of mRNA transcripts, stimulating translational suppression or break of the target $[6,7]$. Multiple thousands of miRNAs have been classified in humans and they are evolutionarily conserved. Lately, it has been uncovered that variable expression of special miRNA genes could be increased during the beginning and improvement of cancer [8]. Accordingly, cancer-associated alterations in miRNA expression models are emerging as promising diagnostic markers often compared with disease improvement and survival rates, thus offering a new window for treatment of another cancer types [9]. In general, miRNAs can control several processes, including metabolism, cell differentiation, proliferation and survival, inflammation, genome stability, tumor invasion and angiogenesis [10]. Appearing in the cell nucleus, miRNA operation on the regulation of gene expression continues in the cytoplasm by binding to its supplementary base-sequence of the targeted mRNA molecule. Subsequently, gene silenc-

\footnotetext{
Submitted: November 21, 2016.

*Correspondence: E-mail: ghorbian20@yahoo.com Abbreviations used: 3'-UTR - 3'-untranslated region; BC - breast cancer; BCC - basal cell carcinoma; DGCR8 - DiGeorge syndrome critical region 8; HCC - hepatocellular carcinoma; miRNAs - microRNAs; PACT - PKR activating protein; P-bodies - processing bodies; PCMM - primary cutaneous malignant melanoma; piRNAs - Piwi-interacting RNAs; pre-miRNAs - precursor miRNAs; pri-miRNAs - primary miRNAs; RISC - RNA-induced silencing complex; SCC - squamous cell carcinoma; siRNAs - small interfering RNAs; TRBP - transactivation-responsive RNA-binding protein; XPO5 - exportin-5.
}

ing through degradation of target mRNA or inhibition with the translation process takes its place [11]. Presently hundreds of miRNAs have been distinguished in different species [12]. Notwithstanding, most miRNA research is focused on the growth and progress of stem cells, differentiation, tumorigenesis and other pathological processes [13].

\section{MIRNA BIOGENESIS AND MECHANISM OF ACTION}

Mainly, miRNAs are encoded within the genome and are transcribed by RNA polymerase II (Pol II) or RNA polymerase III (Pol III) as long precursor transcripts, known as primary miRNAs (pri-miRNAs) of several kilobases $(\mathrm{kb})$ in length [14]. Mature miRNAs are produced from pri-miRNAs by sequential processing strategies. Conventionally animal pri-miRNAs possess nearly 33 bp stem and have a terminal loop structure with flanking segments [15]. Precursor miRNAs (pre-miRNAs) are commonly the microprocessor system in the nucleus, whose core components are the RNase III enzyme Drosha and its binding partner DiGeorge syndrome critical region 8 (DGCR8) [16]. Approximately $31 \%$ of miRNAs are prepared from introns of protein-coding genes, whereas many other miRNAs are expressed from committing miRNA gene loci. An own pri-miRNA can either produce a single miRNA or contain clusters of two or more miRNAs that are changed from a regular primary transcript [17]. However, these long pri-miRNAs are cleaved by Microprocessor, comprising the double-stranded RNase III enzyme Drosha and its crucial cofactor, the doublestranded RNA binding protein DGCR8 [18]. In this pathway, the nuclear splicing machinery supplies pre-miRNA from introns. miRNAs originated from this process are appropriately termed mirtrons. These molecules enclose a short class of miRNAs, but are identified in multiple organisms [19]. Preliminary, the nuclear microprocessor complex identifies the miRNA hairpins in the main transcript and cleaves each hairpin roughly 11 nucleotides from its base [20]. Recent manifestation suggests that some miRNAs that survive 
within introns, the so-called mirtrons, bypass Drosha break and depend on the proceeding of the pre-mRNA splicing machinery to originate an approximately $60 \mathrm{nt}$ pre-miRNA hairpin [21]. Regardless of whether the pre-miRNA hairpin is excised from the initial transcript by canonical Drosha break or through the mirtron pathway, the next stage in the miRNA biogenesis is recognition of the nearly 60 nt pre-miRNA by exportin-5 (XPO5) and export to the cytoplasm in a ran guanine triphosphatase-dependent manner [22]. Mirtrons encompass a small cluster of miRNAs, but are found in numerous organisms. Pre-miRNAs are transported from the nucleus to the cytoplasm by the exportin-5/RanGTPheterocomplex [23]. Complex in cytoplasm compromised of Dicer RNase III endonuclease, transactivation-responsive RNA-binding protein (TRBP), and protein kinase Re-activating. Protein further cleaves the pre-miRNA generating a short double-stranded miRNA:miRNA complex intermediate [24]. In the cytoplasm, pre-miRNAs are processed by RNase III enzyme Dicer. Dicer is thought to react with dsRBDs-containing partner proteins, HIV TRBP and/or PKR activating protein (PACT) [25]. Then, Dicer cleaves pre-miRNAs into 21-25 nt long miRNA/ miRNA duplexes, each strand of which bears 5 ' monophosphate, 3' hydroxyl group and a 3' 2-nt overhang. It preferentially incorporates one of the duplex strands into the RNA-induced silencing complex (RISC). Of a miRNA/miRNA duplex, only one strand, designated the miRNA strand, is selected as the guide of mature RISC, whereas the other strand, the miRNA strand, is discarded during RISC assembly [26]. Such biased strand selection depends on the stability of at least three properties of a miRNA/miRNA duplex: the structure; the 5' nucleotide identity; and the thermodynamic asymmetry [27]. These findings propose miRNA processing by Dicer, during RISC loading, and target RNA cleavage by argonaute 2 (Ago2) is coupled [28]. The translational repression mechanism by miRNAs has been poorly understood. Recently, it was declared that the target mRNAs binding to RISC through partial base pairing, are accumulated in the cytoplasmic foci referred to as processing bodies (P-bodies). P-bodies, in which the mRNAs are stored or degraded by the decapping enzymes and exonucleases, do not contain the translational machinery [29] (Figure).

\section{DICER}

Dicer, a critical RNase III endonuclease of the miRNA processing, performs a role in carcinogenesis and various types of human malignancies [30]. Dicer is a large multi-domain protein with a principal function for the last step of miRNA and short-interfering RNA biogenesis. In human and mouse cell lines, Dicer is considered to act in the nuclear clearance of dsRNA as well as the foundation of chromatin agreements [31]. Dicer is supposed to play an essential function in the biogenesis of eukaryotic small RNAs/ miRNAs; Dicer target transcripts have not been directly mapped. Interestingly, mainly Dicer-binding sites

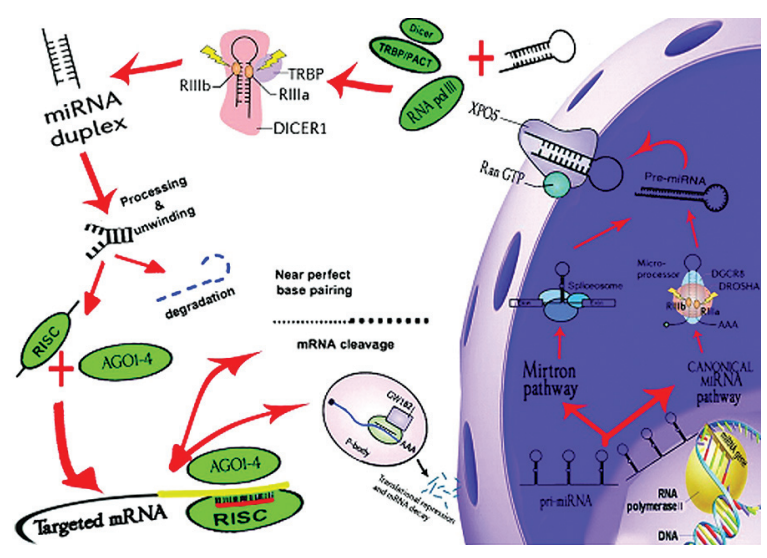

Figure. Schematic presentation of the miRNA biogenesis and functional mechanisms. The miRNA genes are transcribed by RNA polymerase II (Pol II) as long precursor transcripts (pri-miRNAs). These long pri-miRNAs are cleaved by RNase III enzyme Drosha and DGCR8. Pre-miRNAs are exported from the nucleus to the cytoplasm by the XPO5/RanGTP heterocomplex. Complex in cytoplasm compromised of Dicer RNase III endonuclease and TRBP. Protein further cleaves the pre-miRNA generating a short double-stranded miRNA:miRNA complex intermediate. miRNA/miRNA duplex, only one strand, designated the miRNA strand, is selected as the guide of mature RISC and target RNA cleavage by AGO2 is coupled

remain on mRNAs/IncRNAs and are not substantially prepared into miniature RNAs. These passive sites normally harbor short, Dicer-bound hairpins with entire transcripts and regularly maintain target expression [32]. Dicer, single of the proteins implicated in the synthesis miRNA, is implicated in the biogenesis of miRNAs and is influential in carcinogenesis and cancer progress and progression [33]. Low expression of Dicer gene and protein linked to poor prognosis and recurrence of cervical cancer.

In addition, the investigation revealed that decreased Dicer gene expression and protein levels are associated with metastasis relapse and tumor stage. The patients with decreased Dicer miRNA and protein expression displayed a shorter 5-year disease-free survival and overall survival. Moreover, low expression of Dicer befits to be a significant prognostic factor for cervical malignancy and other tumor types [34]. Previous studies have shown that inhibition of Dicer in von Hippel - Lindau deficient clear cell renal cell carcinoma contributed to the high levels of the hypoxia-inducible factors- $2 \alpha$ and a cancer phenotype, which suggests Dicer could be a useful therapeutic target for managing this disease [35]. miR-200a and miR-31 are targeted for Dicer and are involved in the carcinogenesis, cell migration, and behavior of castration-resistant prostate cancer, showing that they could be possible biomarkers for monitoring of prostate cancer progression [36]. The Dicer protein expression is significantly associated with hormone receptor status and cancer subtype in breast tumors [37]. Drosha and Dicer1 mutations impair expression of tumor-suppressing miRNAs, including the let-7 family, significant regulators of MYCN, LIN28 and other Wilms tumor oncogenes. Current findings explored the mechanisms through which mutations in miRNA biogenesis components reprogrammed miRNA expres- 
sion in human malignancies and proposed that these defects clarify a distinct subclass of Wilms tumors [38]. However, several investigations have revealed that dysregulation of Dicer gene are observed in many disorders, including upregulation in Dicer1 gene expression with tumor stages and progression of prostate cancer and smooth muscle tumors [39]. In addition, preliminary investigations revealed that upregulation of Dicer1 is correlated with gastric tumor subtype and advanced tumor stages in gastric cancer and serous ovarian cancer $[40,41]$. However, the other studies showed that the increased Dicer1 expression levels are required for proliferation of oral cancer cells [42]. Faber et al. [43] evaluated Dicer1 expression levels in colorectal cancer, and revealed an increased level, which is associated with tumor stage and poor survival of the patients. Chiosea et al. [44] disclosed that upregulation of Dicer1 expression levels are associated with histological subtypes and stages of lung cancer. Ma et al. [45] showed that up-regulated Dicer1 expression levels correlated with clinical stage of cutaneous melanoma. In general, Dicer expression levels are different in various cancer types; Witkowski et al. [46] revealed that downregulation of Dicer1 expression levels is associated with the altered miRNA profile in patients with bladder cancer. Pampalakis et al. [47] revealed that decreased Dicer1 expression levels are associated with advanced tumor stage and poor survival of ovarian cancer patients. Contradictory to the other findings, Torres et al. [48] showed that downregulation of Dicer 1 gene is not associated with histological grade in patients with endometrial cancer. Guo et al. [49] observed the decreased expression of Dicer1, which correlated with lower survival of patients with nasopharyngeal carcinoma. In addition, Lin et al. [50] revealed that Dicer1 expression levels were significantly lower and associated with total downregulation of miRNAs and poor outcome of patients with neuroblastoma. Khoshnaw et al. [51] revealed that decreased Dicer1 expression levels are associated with breast cancer (BC) progression and recurrence. Furthermore, investigations displayed that decrease of Dicer1 levels are correlated with metastasis, invasion and poor prognosis and shortened survival in the patients with gallbladder adenocarcinoma and non-small cell lung cancer [52, 53]. Wu et al. [54] revealed that downregulation of Dicer1 is not associated with clinical characteristics of hepatocellular carcinoma (HCC). Findings in other studies revealed that decreased Dicer1 expression levels are associated with progression, tumor stage, prognosis and shorter survival of patients with chronic lymphocytic leukemia, colorectal cancer, BC, and papillary thyroid carcinoma (Table) [55-59].

\section{DROSHA}

Drosha involves two RNase III domains, which performs a vital role in miRNA biogenesis; Drosha and its double-stranded RNA-binding partner protein Pasha/DGCR8 likely identify and cut miRNA precursor
RNAs or pri-miRNA hairpins co-transcriptionally [60]. Long pri-miRNAs are cleaved by microprocessor complex comprising of Drosha, a type III ribonuclease (RNase III), and an RNA-binding protein DGCR8, is so called because a Drosha/DGCR8 complex is essential and sufficient to process a pri-miRNA into a pre-miRNA hairpin in vitro. Dicer then cleaves the pre-miRNA hairpin at the loop to form the mature miRNA. Correct Drosha cleavage in the pri-miRNA is critical to the production of a functional miRNA [61]. Drosha is an important factor for miRNA biogenesis and as such obligatory for cellular homeostasis and developmental processes. Together with its cofactor DGCR8, it changes the pri-miRNA into the pre-miRNA in the nucleus. Whilst the middle and the C-terminal domain are important for pri-miRNA processing and DGCR8 binding, the activity of the $\mathrm{N}$-terminus remains mysterious. Different investigations have linked this region to the subcellular localization of Drosha, stabilization, and response to tension [62]. The repression occurs solely in mature miRNAs and not in pri-miRNA transcripts, evidencing that the Drosha E1147K mutation affects processing of pri-miRNAs. The pivotal role of the miRNA biogenesis pathway is shown in Wilms tumor development, particularly the major miRNA processing gene Drosha [63]. Decreased expression of Drosha was found in melanoma. Furthermore, the irregular subcellular location of Drosha reveals potential deregulation in the procedures responsible for its proper localization in the nucleus [64]. The new results show that cytoplasmic Drosha potentially plays a role in blocking carcinogenesis and progression of gastric cancer and may serve as an independent prognostic marker [65]. The copy number discrepancy of Dicer1 and Drosha correlates well with their expression levels and survival of patients with non-small cell lung cancer and other cancer types. The increased expression of Drosha and Dicer1 are associated with decreased and increased survival, respectively. As a result, copy number variation may be a significant mechanism of upregulation/downregulation of miRNAs in malignancy and propose an oncogenic role for Drosha [66]. Muralidhar et al. [39] revealed that upregulation of Drosha expression levels could alter miRNA profile associated with neoplastic progression in cervical squamous cell carcinoma (SCC). In addition, Sugito et al. [67] have shown that increased Drosha expression was associated with poor survival of patients with esophageal cancer. The findings of several investigations have shown that upregulation of Drosha was associated with tumor progression, advanced tumor stages and poor prognosis in the patients with various cancer types [37-39, 51]. Moreover, Sand et al. [68] revealed that upregulation of Drosha expression levels was not determined in SCC and basal cell carcinoma (BCC). Avery-Kiejda et al. [69] revealed that upregulation of Drosha expression levels had no significant clinical correlation in triple-negative BC. Similarly, in others investigations, findings showed that downregulation of Drosha expression levels could 
alter miRNA profile and correlated with poor patient survival, histological grade, metastasis, invasion and poor prognosis in many cancer types [48-50, 52, 64, $70,71]$. Findings revealed that altered Drosha expression levels are not associated with clinical features in colorectal cancer, BC, and papillary thyroid carcinoma (see Table) [57-59].

\section{DGCR8}

Mirtrons pathways include a small class of miRNAs; although miRNAs are synthesized via the miRtron pathway rather than by Drosha, the synthesis of main miRNAs looks to be Drosha dependent. The prominent stem-loop in pri-miRNAs is identified through Drosha together with its partner Pasha/DGCR8. Indeed, Pasha/DGCR8 is thought to bind preferentially at the junction between the stem and the more inflexible loop, and this process can be co-transcriptional. This binding then positions Drosha midway up to the stem so that it is correctly positioned to make a pair of staggered breaks to generate the nearly 70 bp premiRNA [72]. The microprocessor complex mediates intranuclear biogenesis of pre-miRNAs from the primiRNA transcript. Extranuclear, mature miRNAs are merged into the RISC before interaction with completing target mRNA that leads to repression of translation

Table. Patterns of miRNA expression in different tumor types

\begin{tabular}{|c|c|c|c|}
\hline Genes & Increase or decrease & Cancer type & Refs \\
\hline \multirow[t]{17}{*}{ Drosha } & Altered miRNA profile; associated with neoplastic progression & Cervical SCC & [39] \\
\hline & Regulates cell proliferation; associated with poor patient survival & Oesophageal cancer & {$[67]$} \\
\hline & Associated with pathological characteristics and patient survival & Gastric cancer & {$[40]$} \\
\hline & Associated with advanced tumor stages & Serous ovarian carcinoma & [41] \\
\hline & Associated with poor prognosis & Non-small cell lung cancer & {$[53]$} \\
\hline & Not determined & SCC and BCC & [68] \\
\hline & No clinical correlation & Triple-negative BC & [69] \\
\hline & Altered miRNA profile & Bladder cancer & [70] \\
\hline & Associated with poor patient survival & Ovarian cancer & [71] \\
\hline & Correlated with histological grade & Endometrial cancer & {$[48]$} \\
\hline & Correlated with shorter patient survival & Nasopharyngeal carcinoma & [49] \\
\hline & Correlated with metastasis, invasion and poor prognosis & Gallbladder adenocarcinoma & {$[50]$} \\
\hline & Correlated with total downregulation of miRNAs and poor outcome & Neuroblastoma & {$[52]$} \\
\hline & Associated with cancer progression and poor survival & Cutaneous melanoma & {$[64]$} \\
\hline & Down-regulated & $\mathrm{BC}$ & [58] \\
\hline & Significantly upregulated, not associated with clinical characteristics & Colorectal cancer & {$[57]$} \\
\hline & Significantly lower expressed, not associated with clinical characteristics & Papillary thyroid carcinoma & [59] \\
\hline \multirow[t]{21}{*}{ Dicer } & Correlated with a gastric tumor subtype & & \\
\hline & & Gastric cancer & {$[40]$} \\
\hline & Associated with advanced tumor stages & Serous ovarian carcinoma & [41] \\
\hline & Required for proliferation & Oral cancer & {$[42]$} \\
\hline & Correlated with tumor stage and associated with poor survival & Colorectal cancer & {$[43]$} \\
\hline & Associated with histological subtypes and stages & Precursor lesions of lung adenocarcinoma & {$[44]$} \\
\hline & Correlated with clinical stage & Cutaneous melanoma & {$[45]$} \\
\hline & Altered miRNA profile & Bladder cancer & {$[46]$} \\
\hline & Associated with advanced tumor stage and poor patient survival & Ovarian cancer & {$[47]$} \\
\hline & No association with histological grade detected & Endometrial cancer & [48] \\
\hline & Correlated with shorter patient survival & Nasopharyngeal carcinoma & [49] \\
\hline & Associated with a total down regulation of miRNAs and poor outcome & Neuroblastoma & {$[50]$} \\
\hline & Associated with cancer progression and recurrence & $\mathrm{BC}$ & {$[51]$} \\
\hline & Correlated with metastasis, invasion and poor prognosis & Gallbladder adenocarcinoma & {$[52]$} \\
\hline & Low levels of DICER1 expression correlate with shortened survival & Non-small cell lung cancer & [53] \\
\hline & Not associated with clinical characteristics & $\mathrm{HCC}$ & {$[54]$} \\
\hline & Associated with progression and prognosis & Chronic lymphocytic leukemia & {$[55]$} \\
\hline & Associated with tumor stage and shorter survival & Colorectal cancer & {$[56]$} \\
\hline & Significantly upregulated, not associated with clinical characteristics & Colorectal cancer & {$[57]$} \\
\hline & $\begin{array}{l}\text { Significantly altered gene expression, not associated with clinical char- } \\
\text { acteristics }\end{array}$ & Papillary thyroid carcinoma & [59] \\
\hline & Down-regulated & $\mathrm{BC}$ & [58] \\
\hline \multirow[t]{7}{*}{ DGCR8 } & Associated with poor patient survival & Oesophageal cancer & {$[67]$} \\
\hline & Altered miRNA profile & Bladder cancer & {$[70]$} \\
\hline & Not determined & SCC and BCC & [68] \\
\hline & Associated with dysregulated miRNA & Prostate cancer & {$[64]$} \\
\hline & Not associated with any clinical parameters & Colorectal carcinoma & [73] \\
\hline & Required for cell proliferation, migration and invasion & Ovarian cancer & {$[75]$} \\
\hline & $\begin{array}{l}\text { Significantly altered gene expression, not associated with clinical char- } \\
\text { acteristics }\end{array}$ & Papillary thyroid carcinoma & [59] \\
\hline & Associated with an altered miRNA profile & Bladder cancer & [70] \\
\hline \multirow[t]{4}{*}{ AG01/AGO2 } & Not determined & Actinic keratoses, SCC and BCC & [68] \\
\hline & Associated with advanced tumor stages & Serous ovarian carcinoma & [41] \\
\hline & $\begin{array}{l}\text { Correlated with advanced tumor stages and associated with shorter sur- } \\
\text { vival }\end{array}$ & & \\
\hline & Significantly lower expressed, not associated with clinical characteristics & Papillary thyroid carcinoma & [59] \\
\hline \multirow[t]{6}{*}{$T R B P$} & Amplified in BC & $\mathrm{BC}$ & {$[85]$} \\
\hline & Significantly up-regulated, associated with clinical characteristics & $\mathrm{BC}$ & {$[87]$} \\
\hline & Significantly up-regulated & Malignant melanoma & {$[88]$} \\
\hline & Significantly up-regulated & Diffuse large B cell lymphomas & {$[96]$} \\
\hline & $\begin{array}{l}\text { Significantly up-regulated, not associated with histopathological and } \\
\text { clinical parameters }\end{array}$ & Adrenocortical carcinoma & [97] \\
\hline & Altered miRNA profile & Ewing sarcoma & [98] \\
\hline
\end{tabular}

Notes: AGO - Argonaute; BC - breast cancer; BCC - basal cell carcinoma; DGCR8 - DiGeorge syndrome critical region 8; SCC - squamous cell carcinoma; XPO5 - exportin-5; TRBP - transactivation-responsive RNA-binding protein. 
or mRNA destabilization. The DGCR8 is a component of microprocessor complex crucial for miRNA maturation. The Ago2 proteins are a component of a complex protein named as RISC. A previous investigation has shown that DGCR8 mRNA expression is down-regulated in prostate cancer. Upregulated DGCR8 mRNA expression has been found in epithelial skin cancer and pleomorphic adenomas of the salivary gland. It has been documented that the Ago2 mRNA expression level is up-regulated in epithelial skin cancer [73]. Firstly, the DGCR8 mRNA expression level was up-regulated in colorectal cancer, evidencing on its role in the pathobiology of the colorectal carcinogenesis [74]. DGCR8 was expressed in ovarian cancer. MiR-27b was detected as significantly down-regulated miRNA in DGCR8-knockdown cells and endorsed cell proliferation in ovarian cancer cells [75]. Preliminary studies reported that expression levels of Drosha in AGS and HepG2 cell lines were higher than in the controls, whereas, Drosha expression level in KYSE-30 cell line was lower. The Dicer expression levels in AGS and HepG2 cells were increased, whereas, its expression level in KYSE-30 cell was lower. The DGCR8 expression levels in all three cell lines were significantly higher than in the control samples [76]. In addition, Sugito et al. [67] revealed that upregulation of DGCR8 expression was associated with poor survival of patients with esophageal cancer. Catto et al. [70] showed that increased DGCR8 expression levels altered the miRNA profile in bladder cancer. Sand et al. [68] reported that upregulation of DGCR8 expression was not determined in SCC and bladder cancer, additionally, revealed that up-regulated DGCR8 expression was associated with dysregulated miRNA in prostate cancer. Kim etal. [73] revealed that upregulation of DGCR8 expression was not associated with any clinical parameters in colorectal carcinoma. Furthermore, Guo et al. [49] revealed that increased DGCR8 expression levels were required for cell proliferation, migration, and invasion in ovarian cancer. Findings revealed that altered DGCR8 expression is not associated with clinical features in papillary thyroid carcinoma [59].

\section{AGO}

Pre-miRNAs exported into the cytoplasm are processed by another RNase III enzyme, Dicer, into nearly 21 bp double-stranded miRNA-miRNA duplexes and moved into the groove of AGO. Afterwards the miRNA strand dissociation, mature single-stranded miRNA remains loaded into and stabilized by AGO1. AGO was subsequently separated from Dicer to bind TNRC64, an essential co-factor in the miRNA-induced silencing complex [77]. AGO2 is a major part of the RISC that can directly deteriorate mRNA through slicing AGO2 amasses in cytoplasmic processing bodies, where additional binding interactions promote translational inhibition and mRNA decay. AGO2 also couples with MVEs in structures that have been called "GWbodies" because of the presence of GW182 but lack of other P-body components. Current reports have demonstrated that AGO2 binds to miRNAs to generate AGO2-miRNA complexes that are found in the extracellular space. Although the majority of descriptions illustrates Ago2 as being present in the extracellular space as a free protein, other investigations have shown that AGO2 and other RNA-processing proteins are present in the secreted exosomes [78]. The genetic polymorphism of AGO2 may be a risk factor for the progressive lymph node metastasis in nasopharyngeal carcinoma in the Chinese population, and AGO2 acts as an oncogene in the development of nasopharyngeal carcinoma [79]. Sand et al. [68] revealed that upregulation of AGO1 and AGO2 expression was not determined in actinic keratosis, SCC and $\mathrm{BCC}$, while such upregulation was associated with advanced tumor stages in serous ovarian carcinoma. Findings revealed that the AGO2 expression levels was significantly lower in neoplastic tissues compared to the healthy tissues in papillary thyroid carcinoma (see Table) [59].

\section{XPO5}

XPO5 is a component of the importin- $\beta$ family of proteins that consist of one major class of nucleocytoplasmic transporters. XPO5 binds directly to its pre-miRNA cargo in a Ran-GTP-dependent manner. As well, XPO5 is capable of recognizing and export structured RNAs that are unrelated to pre-miRNAs, involving viral mini-helix RNA and tRNA, along with certain other proteins, such as STAU2, ILF3, and $\mathrm{JAZ}$. It has also been indicated that XPO5 is important in siRNA biogenesis and therefore, is a basic point of intersection between the siRNA and miRNA pathways [80]. XPO5 is a transporter protein regularly mediating pre-miRNAs nuclear export. Recent investigations have displayed that XPO5 may play an important role in some cancers. Anyway, little is known about XPO5 in HCC [81]. The XPO5 genetic defect traps pre-miRNAs in the nucleus decreases miRNA processing and diminishes miRNA-target inhibition [82]. XPO5 knockdown promoted $\mathrm{HCC}$ cell migration and decreased the expression of E-cadherin and p53. Furthermore, after treatment with DAC and TSA, the mRNA level of XPO5 was up-regulated in HCC cells, implicating that epigenetic modulation may be involved in the transcription of XPO5. Generally, these findings suggest that XPO5 functions as a potential tumor suppressor in the development and progression of HCC as well as a promising molecular target for HCC therapy [83]. Catto et al. [70] revealed that upregulation of XPO5 expression was associated with the altered miRNA profile in bladder cancer (see Table).

\section{TRBP}

miRNAs are transported from the nucleus to the cytoplasm via XPO5 mediated pathway, where they are bound with Dicer and the TRBP, and maturate into double-stranded miRNAs. The active strand of such mature miRNA is retained in the Dicer-TRBP complex, which then binds with the endonuclease AGO2. One strand of the mature miRNA (the guide strand) 
is loaded into the RISC target mRNAs that are complementary to the miRNA [84]. Huang et al. [85] revealed that TRBP is overexpressed in BC. TRBP is multifunctional and mediates crosstalk between different pathways. The protein AIB3, also known as ASC-2, RAP250, PRIP, TRBP, and NCR, is a newly recognized nuclear receptor co-activator that is amplified and over-expressed in BC [86]. Lin et al. [87] revealed that the $\mathrm{BC}$ patients with cytoplasmic overexpression of TRBP2 had shorter disease free survival and overall survival. Sand et al. [88] showed that TRBP2 was significantly up-regulated in benign melanocytic nevi compared to primary cutaneous malignant melanoma (see Table). Earlier, we have applied a gold standard method for lymphoma diagnosis using TRBP2 gene expression analysis [89-95]. However, Caramuta et al. [96] revealed that the expression of TRBP2 was significantly higher in diffuse large B cell lymphomas than in lymph nodes, and also in the adrenocortical carcinomas compared with adenomas or normal adrenal cortices. Whereas, TRBP2 expression was not correlated with histopathological and clinical parameters [97]. De Vito et al. [98] revealed that deregulation of TRBP2 in the Ewing sarcoma.

In conclusion, the newest findings on miRNA expression patterns in cancerous tissues will allow to develop the use of these molecules as novel biomarkers for tumor diagnosis, prognosis, and therapy.

\section{CONFLICT OF INTERESTS}

The authors have declared no conflict of interests.

\section{REFERENCES}

1. Poursadegh Zonouzi A, Poursadegh Zonouzi A, Ghorbian S. PiRNAs interacting proteins, candidate molecular marker for evaluation of idiopathic male infertility. Andrologia 2014; 46: 823-3.

2. Papaioannou MD, Nef S. microRNAs in the testis: building up male fertility. J Androl 2010; 31: 26-33.

3. He Z, Kokkinaki M, Pant D, et al. Small RNA molecules in the regulation of spermatogenesis. Reproduction 2009; 137: 901-11.

4. Ghorbian S, Poursadegh Zonouzi A. Micro-RNAs in IVF outcome. Indian J Hum Gene 2013; 19: 273.

5. Melo SA, Sugimoto H, O'Connell JT, et al. Cancer exosomes perform cell-independent microRNA biogenesis and promote tumorigenesis. Cancer Cell 2014; 5: 707-21.

6. Chan SP, Slack FJ. And now introducing mammalian mirtrons. Dev Cell 2007; 13: 605-7.

7. Ghorbian S. Micro-RNAs, next-generation molecular markers in male infertility field. Transl Androl Urol 2012; 1: $245-6$.

8. Tsuchiya S, Okuno Y, Tsujimoto G. MicroRNA: biogenetic and functional mechanisms and involvements in cell differentiation and cancer. Pharmacol Sci 2006; 101: 267-70.

9. Kushlinskii NE, Fridman MV, Braga EA. Molecular mechanisms and microRNAs in osteosarcoma pathogenesis. Biochemistry (Mosc) 2016; 81: 315-28.

10. Lin S, Gregory RI. MicroRNA biogenesis pathways in cancer. Nat Rev Cancer 2015; 15: 321-33.

11. Khazaie Y, Nasr Esfahani MH. MicroRNA and male infertility: apotential for diagnosis. Int J Fertil Steril 2014; 2: $113-8$.
12. Chen K, Song F, Calin GA, et al. Polymorphisms in microRNA targets: a gold mine for molecular epidemiology. Carcinogenesis 2008; 29: 1306-11.

13. Liu T, Cheng W, Gao Y, et al. Microarray analysis of microRNA expression patterns in the semen of infertile men with semen abnormalities. Mol Med Rep 2012; 6: 535-42.

14. Ohtsuka M, Ling H, Doki Y, et al. MicroRNA processing and human cancer. J Clin Med 2015; 4: 1651-67.

15. Tomasetti M, Santarelli L, Neuzil J, et al. MicroRNA regulation of cancer metabolism: role in tumour suppression. Mitochondrion 2014; 19: 29-38.

16. Manikandan J, Aarthi JJ, Kumar SD, et al. Oncomirs: the potential role of non-coding microRNAs in understanding cancer. Bioinformation 2008; 2: 330-4.

17. Güttler T, Görlich D. Ran-dependent nuclear export mediators: a structural perspective. EMBO J 2011; 30: $3457-74$

18. Arteaga-Vázquez M, Caballero-Pérez J, Vielle-Calzada JP. A family of microRNAs present in plants and animals. Plant Cell 2006; 18: 3355-69.

19. Bohnsack MT, Czaplinski K, Gorlich D. Exportin 5 is a RanGTP-dependent dsRNA-binding protein that mediates nuclear export of pre-miRNAs. RNA 2004; 10: 185-91.

20. Yang $M$, Chen $Y$, Chen $L$, et al. miR-15b-AGO2 play a critical role in HTR8/SVneo invasion and in a model of angiogenesis defects related to inflammation. Placenta 2016; 41: 62-73.

21. Singh R, Ramasubramanian B, Kanji S, et al. Circulating microRNAs in cancer: hope or hype? Cancer Lett 2016; 381: $113-21$.

22. Alizadeh S, Azizi SG, Soleimani M, et al. The role of microRNAs in myeloproliferative neoplasia. Int J Hematol Oncol Stem Cell Res 2016; 10: 172-85.

23. Soifer HS, Rossi JJ, Saetrom P. MicroRNAs in disease and potential therapeutic applications. Mol Ther 2007; 15: 2070-9.

24. Nelson KM, Weiss GJ. MicroRNAs and cancer: past, present, and potential future. Mol Cancer Ther 2008; 7: 365560.

25. Vettori S, Gay S, Distler O. Role of microRNAs in fibrosis. Open Rheumatol 2012; 6: 130-9.

26. Zhang PY, Li G, Deng ZJ, et al. Dicer interacts with SIRT7 and regulates H3K18 deacetylation in response to DNA damaging agents. Nucl Acids Res 2016; 44: 3629-42.

27. Kwak PB, Iwasaki S, Yukihide T. The microRNA pathway and cancer. Cancer Sci 2010; 101: 2309-15.

28. Chen M, Calin GA, Meng QH. Circulating microRNAs as promising tumor biomarkers. Adv Clin Chem 2014; 67: 189-214.

29. Martinez NJ, Gregory RI. Argonaute 2 expression is post-transcriptionally coupled to microRNA abundance. RNA 2013; 19: 605-12.

30. Zhang LI, Wang C, Liu S, et al. Prognostic significance of Dicer expression in hepatocellular carcinoma. Oncol Lett 2016; 11: 3961-6.

31. Much C, Auchynnikava T, Pavlinic D, et al. Endogenous mouse Dicer is an exclusively cytoplasmic protein. PLoS Genet 2016; 12: e1006095.

32. Rybak-Wolf A, Jens M, Murakawa Y, et al. A variety of dicer substrates in human and C. elegans. Cell 2014; 159: $1153-67$.

33. Yu YY, Kuang D, Yin XX. Association between the DICER rs1057035 polymorphism and cancer risk: evidence from a meta-analysis of 1,2675 individuals. Asian Pac J Cancer Prev 2015; 16: 119-24. 
34. He L, Wang HY, Zhang L, et al. Prognostic significance of low DICER expression regulated by miR-130a in cervical cancer. Cell Death Dis 2014; 5: e1205.

35. Fan Y, Li H, Ma X, et al. Dicer suppresses the malignant phenotype in VHL-deficient clear cell renal cell carcinoma by inhibiting HIF-2 $\alpha$. Oncotarget 2016; 7: 18280-94.

36. Bian X, Shen $Y$, Zhang G, et al. Expression of dicer and its related miRNAs in the progression of prostate cancer. PLoS One 2015; 10: e0120159.

37. Grelier G, Voirin N, Ay AS, et al. Prognostic value of Dicer expression in human breast cancers and association with the mesenchymal phenotype. Br J Cancer 2009; 101: 673-83.

38. Rakheja D, Chen KS, Liu Y, et al. Somatic mutations in DROSHA and DICER1 impair microRNA biogenesis through distinct mechanisms in Wilms tumours. Nat Commun 2014; 2: 4802.

39. Muralidhar B, Winder D, Murray M, et al. Functional evidence that Drosha overexpression in cervical squamous cell carcinoma affects cell phenotype and microRNA profiles. J Pathol 2011; 224: 496-507.

40. Tchernitsa O, Kasajima A, Schäfer R,et al. Systematic evaluation of the miRNA-ome and its downstream effects on mRNA expression identifies gastric cancer progression. J Pathol 2010; 222: 310-9.

41. Vaksman O, Hetland TE, Trope' CG, et al. Argonaute, Dicer, and Drosha are up-regulated along tumor progression in serous ovarian carcinoma. Hum Pathol 2012; 43: 2062-9.

42. Jakymiw A, Patel RS, Deming N, et al. Overexpression of dicer as a result of reduced let-7 MicroRNA levels contributes to increased cell proliferation of oral cancer cells. Genes Chromosomes Cancer 2010; 49: 549-59.

43. Faber C, Horst D, Hlubek F, et al. Overexpression of Dicer predicts poor survival in colorectal cancer. Eur J Cancer 2011; 47: 1414-9.

44. Chiosea S, Jelezcova E, Chandran U, et al. Overexpression of Dicer in precursor lesions of lung adenocarcinoma. Cancer Res 2007; 67: 2345-50.

45. Ma Z, Swede H, Cassarino D, et al. Up-regulated Dicer expression in patients with cutaneous melanoma. PLoS One 2011; 6: e20494.

46. Witkowski L, Mattina J, Schönberger S, et al. DICER1 hotspot mutations in non-epithelial gonadal tumours. Br J Cancer 2013; 109: 2744-50.

47. Pampalakis G, Diamandis EP, Katsaros D, et al. Down-regulation of dicer expression in ovarian cancer tissues. Clin Biochem 2010; 43: 324-7.

48. Torres A, Torres K, Paszkowski T, et al. Major regulators of microRNAs biogenesis Dicer and Drosha are down-regulated in endometrial cancer. Tumour Biol 2011; 32: 769-76.

49. Guo $X$, Liao $Q$, Chen $P$, et al. The microRNAprocessing enzymes: Drosha and Dicer can predict prognosis of nasopharyngeal carcinoma. J Cancer Res Clin Oncol 2012; 138: $49-56$.

50. Lin RJ, Lin YC, Chen J, et al. MicroRNA signature and expression of Dicer and Drosha can predict prognosis and delineate risk groups in neuroblastoma. Cancer Res 2010; 70: $7841-50$.

51. Khoshnaw SM, Rakha EA, Abdel-Fatah TM, et al. Loss of Dicer expression is associated with breast cancer progression and recurrence. Breast Cancer Res Treat 2012; 135: 403-13.

52. Shu GS, Yang ZL, Liu DC. Immunohistochemical study of Dicer and Drosha expression in the benign and malignant lesions of gallbladder and their clinicopathological significances. Pathol Res Pract 2012; 208: 392-7.
53. Díaz-García CV, Agudo-López A, Pérez C, et al. DICER1, DROSHA and miRNAs in patients with non-small cell lung cancer: implications for outcomes and histologic classification. Carcinogenesis 2013; 34: 1031-8.

54. Wu JF, Shen W, Liu NZ, et al. Down-regulation of Dicer in hepatocellular carcinoma. Med Oncol 2011; 28: 804-9.

55. Zhu DX, Fan L, Lu RN, et al. Downregulated Dicer expression predicts poor prognosis in chronic lymphocytic leukemia. Cancer Sci 2012; 103: 875-81.

56. Faggad A, Kasajima A, Weichert W, et al. Downregulation of the microRNA processing enzyme Dicer is a prognostic factor in human colorectal cancer. Histopathology 2012; 61: $552-61$.

57. Kim S, Song ML, Min H, et al. MiRNA biogenesis-associated RNase III nucleases Drosha and Dicer are upregulated in colorectal adenocarcinoma. Oncology Lett 2017; 14: 4379-83.

58. Yan M, Huang H-Y, Wang T, et al. Dysregulated expression of dicer and drosha in breast cancer. Pathol Oncol Res 2012; 18: 343-8.

59. Kim J, Park W-J, Jeong K-J, et al. Racial differences in expression levels of miRNA machinery-related genes, Dicer, Drosha, DGCR8, and AGO2, in Asian Korean papillary thyroid carcinoma and comparative validation using the Cancer Genome Atlas. Int J Genomics 2017; 2017: 5789769.

60. Kadener S, Rodriguez J, Abruzzi KC, et al. Genome-wide identification of targets of the drosha-pasha/ DGCR8 complex. RNA 2009; 15: 537-45.

61. Tu CC, Wng JY. EnABLing microprocessor for apoptosis. Mol Cell Oncol 2016; 3: e1081860.

62. Link S, Grund SE, Diederichs S. Alternative splicing affects the subcellular localization of Drosha. Nucleic Acids Res 2016; 44: 5330-43.

63. Torrezan GT, Ferreira EN, Nakahata AM, et al. Recurrent somatic mutation in DROSHA induces microRNA profile changes in Wilms tumour. Nat Commun 2014; 5: 4039.

64. Jafarnejad SM, Sjoestroem C, Martinka M, et al. Expression of the RNase III enzyme DROSHA is reduced during progression of human cutaneous melanoma. Mod Pathol 2013; 26: 902-10.

65. Zhang H, Hou Y, Xu L, et al. Cytoplasmic Drosha is aberrant in precancerous lesions of gastric carcinoma and its loss predicts worse outcome for gastric cancer patients. Dig Dis Sci 2016; 61: 1080-90.

66. Czubak K, Lewandowska MA, Klonowska K, et al. High copy number variation of cancer-related microRNA genes and frequent amplification of DICER1 and DROSHA in lung cancer. Oncotarget 2015; 6: 23399-416.

67. Sugito N, Ishiguro H, Kuwabara Y, et al. RNASEN regulates cell proliferation and affects survival in esophageal cancer patients. Clin Cancer Res 2006; 12: 7322-8.

68. Sand M, Gambichler T, Skrygan M, et al. Expression levels of the microRNA processing enzymes Drosha and dicer in epithelial skin cancer. Cancer Invest 2010; 28: 649-53.

69. Avery-Kiejda KA, Braye SG, Forbes JF, et al. The expression of Dicer and Drosha in matched normal tissues, tumours and lymph node metastases in triple negative breast cancer. BMC Cancer 2014; 11: 253.

70. Catto JW, Miah S, Owen HC, et al. Distinct microRNA alterations characterize high- and low-grade bladder cancer. Cancer Res 2009; 69: 8472-81.

71. Merritt WM, Lin YG, Han LY, et al. Dicer, Drosha, and outcomes in patients with ovarian cancer. N Engl J Med 2008; 359: 2641-50.

72. Sand M, Skrygan M, Georgas D, et al. Expression levels of the microRNA maturing microprocessor complex 
component DGCR8 and the RNA-induced silencing complex (RISC) components argonaute-1, argonaute-2, PACT, TARBP1, and TARBP2 in epithelial skin cancer. Mol Carcinog 2012; 51: 916-22.

73. Kim B, Lee JH, Park JW, et al. An essential microRNA maturing microprocessor complex component DGCR8 is up-regulated in colorectal carcinomas. Clin Exp Med 2014; 14: 331-6.

74. Roberts TC. The microRNA machinery. Adv Exp Med Biol 2015; 887: 15-30.

75. Guo Y, Tian P, Yang C, et al. Silencing the doublestranded RNA binding protein DGCR8 inhibits ovarian cancer cell proliferation, migration, and invasion. Pharm Res 2015; 32: 769-78.

76. Jafari N, Dogaheh HP, Bohlooli S, et al. Expression levels of microRNA machinery components Drosha, Dicer and DGCR8 in human (AGS, HepG2, and KEYSE-30) cancer cell lines. Int J Clin Exp Med 2013; 6: 269-74.

77. Gibbings D, Mostowy S, Jay F, et al. Corrigendum: selective autophagy degrades DICER and AGO2 and regulates miRNA activity. Nat Cell Biol 2015; 17: 1088.

78. McKenzie AJ, Hoshino D, Hong NH, et al. KRASMEK signaling controls Ago2 sorting into exosomes. Cell Rep 2016; 15: 978-87.

79. Li P, Meng J, Zhai Y, et al. Argonaute 2 and nasopharyngeal carcinoma: a genetic association study and functional analysis. BMC Cancer 2015; 15: 862.

80. Leaderer D, Hoffman AE, Zheng $T$, et al. Genetic and epigenetic association studies suggest a role of microRNA biogenesis gene exportin-5 (XPO5) inbreast tumorigenesis. Int J Mol Epidemiol Genet 2011; 2: 9-18.

81. Kim YK, Kim B, Kim VN. Re-evaluation of the roles of DROSHA, Export in 5, and DICER in microRNA biogenesis. Proc Natl Acad Sci U S A 2016; 113: 1881-9.

82. Melo SA, Moutinho C, Ropero S, et al. A genetic defect in exportin-5 traps precursor microRNAs in the nucleus of cancer cells. Cancer Cell 2010; 18: 303-15.

83. Li Y, Wang X, He B, et al. Downregulation and tumor suppressive role of XPO5 in hepatocellular carcinoma. Mol Cell Biochem 2016; 415: 197-205.

84. Daniels SM, Sinck L, Ward NJ, et al. HIV-1 RRE RNA acts as an RNA silencing suppressor by competing with TRBP-bound siRNAs. RNA Biol 2015; 123: 123-35.

85. Huang JT, Wang J, Srivastava V, et al. MicroRNA machinery genes as novel biomarkers for cancer. Front Oncol 2014; 4: 113.
86. Zhang H, Liao L, Kuang SQ, et al. Spatial distribution of the messenger ribonucleic acid and protein of the nuclear receptor coactivator, amplified in breast cancer-3, in mice. Endocrinology 2003; 144: 1435-43.

87. Lin X, Wu M, Liu P, et al. Up-regulation and worse prognostic marker of cytoplasmic TARBP2 expression in obstinate breast cancer. Med Oncol 2014; 31: 868.

88. Sand M, Skrygan M, Georgas D, et al. The miRNA machinery in primary cutaneous malignant melanoma, cutaneous malignant melanoma metastases and benign melanocytic nevi. Cell Tissue Res 2012; 350: 119-26.

89. Ghorbian S, Jahanzad I, Asghari EM, et al. Molecular analysis of $I G H$ and incomplete $I G H D-J$ clonal gene rearrangement in Hodgkin lymphoma malignancies. Clin Lab 2015; 61: 951-5.

90. Ghorbian S, Jahanzad I, Javadi GR, Sakhinia E. Evaluation of $I G K$ and $I G L$ molecular gene rearrangements according to the BIOMED-2 protocols for clinical diagnosis of Hodgkin lymphoma. Hematology 2016; 21: 133-7.

91. Tohidirad M, Estiar MA, Rezamand A, et al. BCL-1 gene rearrangements in Iranian non-Hodgkin lymphoma patients. Glob J Health Sci 2016; 1: 53396.

92. Kiaei A, Onsori H, Ghorbian S, et al. Detection of $\mathrm{t}(8 ; 14) \mathrm{C}$-MYC/IGH gene rearrangement by long-distance polymerase chain reaction in patients with diffuse large B-cell lymphoma. Hematol Oncol Stem Cell Ther 2016; 9: 141-6.

93. Ghorbian S. Molecular pathology diagnosis of diffuse large B cell lymphoma using BIOMED-2 IGH and IGH D-J clonal gene rearrangements. Ann Diagn Pathol 2017; 29: $28-31$.

94. Moharrami G, Ghorbian S, Seifi M, et al. Detection of immunoglobulin $I G H$ gene rearrangements on formalinfixed, paraffin embedded tissue in lymphoid malignancies. Cell Mol Biol (Noisy-le-Grand) 2014; 60: 43-7.

95. Ghorbian S, Jahanzad I, Javadi GR, et al. Evaluation diagnostic usefulness of immunoglobulin light chains ( $I g \varkappa, I g \lambda)$ and incomplete $I G H$ D-J clonal gene rearrangements in patients with B cell non-Hodgkin lymphomas using BIOMED-2 Protocol. Clin Transl Oncol 2014; 16: 1006-11.

96. Caramuta S, Lee L, Ozata DM, et al. Role of microRNAs and microRNA machinery in the pathogenesis of diffuse large B-cell lymphoma. Blood Cancer J 2013; 3: e152.

97. Caramuta S, Lee L, Ozata DM, et al. Clinical and functional impact of TARBP2 over-expression in adrenocortical carcinoma. Endocr Relat Cancer 2013; 20: 551-64. 